\title{
Spectrum of Discrete Second-Order Neumann Boundary Value Problems with Sign-Changing Weight
}

\author{
Ruyun Ma, Chenghua Gao, and Yanqiong Lu \\ Department of Mathematics, Northwest Normal University, Lanzhou 730070, China \\ Correspondence should be addressed to Ruyun Ma; ruyun_ma@126.com
}

Received 27 May 2013; Accepted 27 July 2013

Academic Editor: Yansheng Liu

Copyright (C) 2013 Ruyun Ma et al. This is an open access article distributed under the Creative Commons Attribution License, which permits unrestricted use, distribution, and reproduction in any medium, provided the original work is properly cited.

\begin{abstract}
We study the spectrum structure of discrete second-order Neumann boundary value problems (NBVPs) with sign-changing weight. We apply the properties of characteristic determinant of the NBVPs to show that the spectrum consists of real and simple eigenvalues; the number of positive eigenvalues is equal to the number of positive elements in the weight function, and the number of negative eigenvalues is equal to the number of negative elements in the weight function. We also show that the eigenfunction corresponding to the $j$ th positive/negative eigenvalue changes its sign exactly $j-1$ times.
\end{abstract}

\section{Introduction}

Let $n>2$ be an integer, $\mathbb{T}=\{1,2, \ldots, n\}$. Let us consider the discrete second-order linear Neumann eigenvalue problem

$$
\begin{array}{r}
\Delta[p(t-1) \Delta u(t-1)]+\lambda m(t) u(t)=0, \\
t \in \mathbb{T}, \\
u(0)=u(1), \quad u(n)=u(n+1),
\end{array}
$$

where $\mathbb{T}=\{1,2, \ldots, n\}, p:\{0,1, \ldots, n\} \rightarrow[0, \infty)$ and $m:$ $\mathbb{T} \rightarrow \mathbb{R}$ satsifies

(A0) $p(j)>0, j \in\{1, \ldots, n-1\}, p(0)=p(n)=0$;

(A1) $m(t) \neq 0$ on $\mathbb{T}$ and $m$ changes its sign on $\mathbb{T}$, that is, there exists a proper subset $\mathbb{T}_{+} \subset \mathbb{T}$, such that

$$
m(t)>0, \quad t \in \mathbb{T}_{+} ; \quad m(t)<0, \quad t \in \mathbb{T} \backslash \mathbb{T}_{+} .
$$

Let $n^{+}$be the number of elements in $\mathbb{T}_{+}$and let $n^{-}$be the number of elements in $\mathbb{T} \backslash \mathbb{T}_{+}$. Then

$$
n^{+}+n^{-}=n
$$

When the weight function $m(t)$ is of one sign, Atkinson [1] and Jirari [2] studied the eigenvalue of the second-order problem

$$
\begin{gathered}
\Delta[p(t-1) \Delta y(t-1)]-q(t) y(t)+\lambda m(t) y(t)=0, \\
t \in \mathbb{T}, \\
y(0)-\alpha y(1)=y(n+1)-\beta y(n)=0,
\end{gathered}
$$

and obtained that (5), (6) have $n$ real eigenvalues, which can be ordered as $\lambda_{1}<\lambda_{2}<\cdots<\lambda_{n}$. Here $q(t) \geq 0$ and $\alpha, \beta \epsilon$ $[0,1]$ is constant. It can be seen that if we take $q(t)=0, \alpha=$ $\beta=1$, then (5), (6) will convert to (1), (2).

However, these two results do not give any information on the sign-changing of the eigenfunction of (5), (6).

In 1991, Kelley and Peterson [3] considered the linear eigenvalue problems (5), (6) with $\alpha=\beta=0$, where $p(t)>0$ on $\{0,1, \ldots n\}, q(t)$ is defined and real valued on $\mathbb{T}$ and $m(t)>$ 0 on $\mathbb{T}$. They obtained that (5), (6) have exactly $n$ real and simple eigenvalues $\lambda_{k}, k \in \mathbb{T}$ which satisfies

$$
\lambda_{1}<\lambda_{2}<\cdots<\lambda_{n}
$$

and the eigenfunction corresponding to $\lambda_{k}$ changes its sign exactly $k-1$ times. 
Furthermore, when $m(t) \equiv 1$, Agarwal et al. [4] generalized the above results to the dynamic equations with SturmLiouville boundary condition. Moreover, under the assumption that the weight functions are of one sign, for further important results in linear Hamiltonian difference systems, including the oscillation properties of solutions, one can see Shi and Chen [5], Bohner [6], and the references therein. The spectrum results for the continuous case have been studied and used to deal with several nonlinear problems; see, for example, [7-13] and the references therein.

However, there are few results on the spectrum of discrete second-order linear eigenvalue problems when $m(t)$ changes its sign on T. In 2007, Ji and Yang [14, 15] studied the structure of the eigenvalues of (5), (6) with $m(t)$ changing its sign, and they obtained the number of positive eigenvalues equal to the number of positive elements in the weight function, and the number of negative eigenvalues equals to the number of negative elements in the weight function. It is worth remaking that they provided no information on the distribution of these eigenvalues of (1), (2) and no information on the signchanging of the corresponding eigenfunctions.

Naturally, there are two interesting questions: (a) how to distribute of the eigenvalues of (1), (2) and (b) how the signchanging of the corresponding eigenfunctions occur.

It is the purpose of this paper to establish the structure of eigenvalues and the oscillatory properties of the corresponding eigenfunctions of (1), (2).

The main result of our paper is the following theorem.

Theorem 1. Suppose that (A0), (A1) hold. Then one has the following.

(i) If $\sum_{t=1}^{n} m(t)>0$, then (1), (2) have $n$ real and simple eigenvalues, which can be ordered as follows:

$$
\lambda_{n^{-}}^{-}<\lambda_{n^{-}-1}^{-}<\cdots<\lambda_{2}^{-}<\lambda_{1}^{-}<0=\lambda_{1}^{+}<\lambda_{2}^{+}<\cdots<\lambda_{n^{+}}^{+} .
$$

Moreover, for $k \in\left\{1, \ldots, n^{-}\right\}$, the eigenfunction $\psi_{k}^{-}$ corresponding to the eigenvalue $\lambda_{k}^{-}$has exactly $k-1$ simple generalized zeros; for $k \in\left\{1, \ldots, n^{+}\right\}$, the eigenfunction $\psi_{k}^{+}$corresponding to the eigenvalue $\lambda_{k}^{+}$has exactly $k-1$ simple generalized zeros. (ii) If $\sum_{t=1}^{n} m(t)<0$, then (1), (2) have $n$ real and simple eigenvalues, which can be ordered as follows:

$$
\lambda_{n^{-}}^{-}<\lambda_{n^{-}-1}^{-}<\cdots<\lambda_{2}^{-}<\lambda_{1}^{-}=0<\lambda_{1}^{+}<\lambda_{2}^{+}<\cdots<\lambda_{n^{+}}^{+} \text {. }
$$

Moreover, for $k \in\left\{1, \ldots, n^{-}\right\}$, the eigenfunction $\psi_{k}^{-}$ corresponding to the eigenvalue $\lambda_{k}^{-}$has exactly $k-$ 1 simple generalized zeros; for $k \in\left\{1, \ldots, n^{+}\right\}$, the eigenfunction $\psi_{k}^{+}$corresponding to the eigenvalue $\lambda_{k}^{+}$ has exactly $k-1$ simple generalized zeros.

(iii) If $\sum_{t=1}^{n} m(t)=0$, then $\lambda=0$ is an eigenvalue of (1), (2) and other $n-2$ eigenvalues are real and simple, which can be ordered as follows:

$$
\lambda_{n^{-}}^{-}<\lambda_{n^{-}-1}^{-}<\cdots<\lambda_{2}^{-}<\lambda_{1}^{-}=0=\lambda_{1}^{+}<\lambda_{2}^{+}<\cdots<\lambda_{n^{+}}^{+} .
$$

Moreover, for $k \in\left\{2, \ldots, n^{-}\right\}$, the eigenfunction $\psi_{k}^{-}$ corresponding to the eigenvalue $\lambda_{k}^{-}$has exactly $k-1$ simple generalized zeros; for $k \in\left\{2, \ldots, n^{+}\right\}$, the eigenfunction $\psi_{k}^{+}$corresponding to the eigenvalue $\lambda_{k}^{+}$has exactly $k-1$ simple generalized zeros.

Remark 2. It is worth remarking that the number of sign changing of eigenfunction is given in Theorem 1. Thus, this result is a generalization of the main results in [15].

Remark 3. Applying Theorem 1 and the well-known Rabinowitz global bifurcation theorem, it is easy to obtain existence results of sign-changing solutions for the nonlinear analogue of (1)-(2); see Ma and Gao [12, 16] for some related results.

The rest of the paper is devoted to proving Theorem 1 . To do this, we make use of the law of inertia for quadratic forms and some techniques form oscillation matrices [17].

\section{Proof of the Main Result}

Let $c(t)=p(t-1)+p(t)$ for $t=2, \ldots, n-1, c(1)=p(1)$, $c(n)=p(n-1)$. Then (1), (2) can be written as a linear pencil problem as follows:

$$
J u=\lambda D u,
$$

where

$$
J=\left(\begin{array}{ccccccc}
p(1) & -p(1) & 0 & \cdots & 0 & 0 & 0 \\
-p(1) & p(1)+p(2) & -p(2) & \cdots & 0 & 0 & 0 \\
0 & -p(2) & p(2)+p(3) & \cdots & 0 & 0 & 0 \\
\vdots & \vdots & \vdots & \ddots & \vdots & \vdots & \vdots \\
0 & 0 & 0 & \cdots & -p(n-2) & p(n-2)+p(n-1) & -p(n-1) \\
0 & 0 & 0 & \cdots & 0 & -p(n-1) & p(n-1)
\end{array}\right)
$$




$$
D=\left(\begin{array}{ccccc}
m_{1} & 0 & \cdots & 0 & 0 \\
0 & m_{2} & \cdots & 0 & 0 \\
\vdots & \vdots & \ddots & \vdots & \vdots \\
0 & 0 & \cdots & m_{n-1} & 0 \\
0 & 0 & \cdots & 0 & m_{n}
\end{array}\right)
$$

Let $J_{j}$ denote the $j$ th principal submatrix of $J$ and $D_{j}$ the $j$ th principal submatrix of $D$. It is easy to verify that $J_{i}$ is positive definite for $i=1, \ldots, n-1, J$ is positive semidefinite and

$$
\operatorname{det} J=0 \text {. }
$$

In fact, for any real vector $\mathbf{x}=\left(x_{1}, x_{2}, \ldots, x_{n-1}\right) \in \mathbb{R}^{n-1}$, it follows that

$$
\mathbf{x} J_{n-1} \mathbf{x}^{T}=\sum_{s=1}^{n-2} p(s)\left(x_{s+1}-x_{s}\right)^{2}+p(n-1) x_{n-1}^{2} \geq 0 .
$$

From (A0), $p(s)>0, s \in\{1,2, \ldots, n-1\}$. If $\mathbf{x} J_{n-1} \mathbf{x}^{T}=0$, then $x_{i}=x_{i+1}$ for $1 \leq i \leq n-1$ and $x_{n-1}=0$, leading to $\mathbf{x}=0$. So, $J_{n-1}$ is positive definite. By the same method, with obvious changes, we can conclude that $J_{i}$ is also positive definite for $j=1,2, \ldots, n-2$.

For any real vector $\mathbf{y}=\left(y_{1}, y_{2}, \ldots, y_{n}\right) \in \mathbb{R}^{n}$, we have $\mathbf{y} J \mathbf{y}^{T}=\sum_{s=1}^{n-1} p(s)\left(y_{s+1}-y_{s}\right)^{2} \geq 0$. Thus, $J$ is positive semidefinite.

For $j=1,2, \ldots, n$, let $Q_{j}(\lambda)$ denote the $j$ th principal subdeterminant of $J-\lambda D$ and suppose that $Q_{0}(\lambda)=1$; then $Q_{n}(\lambda)=\operatorname{det}(J-\lambda D)$, and

$$
\begin{gathered}
Q_{0}(\lambda)=1 ; \\
Q_{1}(\lambda)=c(1)-\lambda m(1) ; \\
Q_{j}(\lambda)=(c(j)-\lambda m(j)) Q_{j-1}(\lambda)-p^{2}(j-1) Q_{j-2}(\lambda), \\
j=2,3, \ldots, n-1 ; \\
Q_{n}(\lambda)=(c(n)-\lambda m(n)) Q_{n-1}(\lambda)-p^{2}(n-1) Q_{n-2}(\lambda),
\end{gathered}
$$

where $c(j)=p(j-1)+p(j), j=2, \ldots, n-1, c(0)=p(1)$ and $c(n)=p(n-1)$.

As we know, to find the eigenvalues of (1), (2) is equivalent to find the roots of $Q_{n}(\lambda)$. Thus, it is necessary to discuss some properties of the sequence (15).

For $j \in\{1, \ldots, n\}$, let $j^{+}$be the number of the elements in $\{m(i) \mid m(i)>0$ for som $i \in\{1, \ldots, j\}\}$, and $j^{-}$the number of the elements in $\{m(i) \mid m(i)<0$ for some $i \in\{1, \ldots, j\}\}$.

Lemma 4. For $j \in\{1, \ldots, n\}$, one has

$$
\begin{aligned}
& \lim _{\lambda \rightarrow-\infty}(-1)^{j^{-}} Q_{j}(\lambda)=+\infty, \\
& \lim _{\lambda \rightarrow+\infty}(-1)^{j^{+}} Q_{j}(\lambda)=+\infty .
\end{aligned}
$$

Proof. For $j \in\{1, \ldots, n\}$, it is evident that $Q_{j}(\lambda)$ is a polynomial of degree precisely $j$, and

$$
Q_{j}(\lambda)=m(1) \cdots m(j)(-\lambda)^{j}+O\left(\lambda^{j-1}\right)
$$

Lemma 5. The roots of $Q_{j}(\lambda)$ are real, $j=1,2, \ldots, n$. Moreover, $Q_{j}(\lambda)=0(j=1,2, \ldots, n-1)$ has $j^{+}$positive roots and $j^{-}$negative roots.

Proof. Since $J_{j}$ is positive definite matrix for $j=1,2, \ldots, n-1$ and $J$ is positive semidefinite matrix, it follows that the roots of $Q_{j}(\lambda)$ are real, $j=1,2, \ldots, n$.

For the $J_{j}, j=1,2, \ldots, n-1$, there exists a unique lower triangular real matrix $L$ such that

$$
L L^{T}=J_{j}
$$

(this is the well-known Cholesky decomposition; see [18, Corollary 7.2.9]). It is easy to check that the matrix $L^{-1} D_{j}\left(L^{T}\right)^{-1}$ is real and symmetric, and $\lambda$ is a root of $Q_{j}(\lambda)$ if and only if $1 / \lambda$ is an eigenvalue of $L^{-1} D_{j}\left(L^{T}\right)^{-1}$.

The fact that $L^{-1} D_{j}\left(L^{T}\right)^{-1}$ is real and symmetric indicates that there exists an orthogonal matrix $Q$ such that

$$
Q^{T} L^{-1} D_{j}\left(L^{T}\right)^{-1} Q=\operatorname{diag}\left(a_{1}, \ldots, a_{j}\right)
$$

where $a_{1} \geq a_{2} \geq \cdots \geq a_{j}$ are all eigenvalues of $L^{-1} D_{j}\left(L^{T}\right)^{-1}$. Let $x=\left(L^{T}\right)^{-1} \mathrm{Q} z$. It is seen from (19) that

$$
\sum_{i=1}^{j} a_{i} z_{i}^{2}=\sum_{i=1}^{j} m(i) x_{i}^{2}
$$

are two representations of the real quadratic form $x^{T} D_{j} x$. In view of the law of inertia for quadratic forms [19, Theorem 1 , p. 297], we immediately deduce that the number of positive and the number of negative elements in the set $\left\{a_{1}, \ldots, a_{j}\right\}$ are $j^{+}$and $j^{-}$, respectively.

Lemma 6. Two consecutive polynomials $Q_{j-1}(\lambda), Q_{j}(\lambda)$ have no common zeros for $j=1, \ldots, n$.

Proof. Suppose on the contrary that there exists $\lambda=\lambda_{0}$ such that $Q_{j-1}\left(\lambda_{0}\right)=Q_{j}\left(\lambda_{0}\right)=0$. Then by the recurrence relation (15), we get $Q_{j-2}\left(\lambda_{0}\right)=0$. Furthermore, we can get $Q_{j-3}\left(\lambda_{0}\right)=\cdots=Q_{1}\left(\lambda_{0}\right)=Q_{0}\left(\lambda_{0}\right)=0$. However, this contradicts $Q_{0}\left(\lambda_{0}\right)=1$. 
Lemma 7. Suppose that $\lambda=\lambda_{0}$ is a root of $Q_{j}(\lambda)$. Then $Q_{j-1}\left(\lambda_{0}\right) Q_{j+1}\left(\lambda_{0}\right)<0$ for $j=1, \ldots, n-1$.

Proof. Since $Q_{j}\left(\lambda_{0}\right)=0$, by the Lemma 6, we have $Q_{j-1}\left(\lambda_{0}\right) \neq 0$. By the recurrence relation $(15), Q_{j+1}\left(\lambda_{0}\right)=$ $-p^{2}(j) Q_{j-1}\left(\lambda_{0}\right)$, which implies that $Q_{j+1}\left(\lambda_{0}\right) Q_{j-1}\left(\lambda_{0}\right)=$ $-p^{2}(i) Q_{j-1}^{2}\left(\lambda_{0}\right)<0$. This completes the assertion.
Lemma 8. Assume that (A0), (A1) hold. Then

(1) $Q_{n}^{\prime}(0)=-p(1) \cdots p(n-1) \sum_{s=1}^{n} m(s)$;

(2) If $\sum_{s=1}^{n} m(s)=0$, then $Q_{n}^{\prime \prime}(0)<0$.

Proof. (1) From (11), we have

$$
\begin{aligned}
& Q_{n}^{\prime}(\lambda)=\frac{d|J u-\lambda D u|}{d \lambda} \\
& =\left|\begin{array}{ccccc}
-m(1) & 0 & \cdots & 0 & 0 \\
-p(1) & p(1)+p(2)-\lambda m(2) & \cdots & 0 & 0 \\
0 & -p(2) & \cdots & 0 & 0 \\
\vdots & \vdots & \ddots & \vdots & \vdots \\
0 & 0 & \cdots & p(n-2)+p(n-1)-\lambda m(n-1) & -p(n-1) \\
0 & 0 & \cdots & -p_{n-1} & p_{n-1}-\lambda m_{n}
\end{array}\right| \\
& +\left|\begin{array}{ccccc}
p(1)-\lambda m(1) & -p(1) & \cdots & 0 & 0 \\
0 & -m(2) & \cdots & 0 & 0 \\
0 & -p(2) & \cdots & 0 & 0 \\
\vdots & \vdots & \ddots & \vdots & \vdots \\
0 & 0 & \cdots & p(n-2)+p(n-1)-\lambda m(n-1) & -p(n-1) \\
0 & 0 & \cdots & -p(n-1) & p(n-1)-\lambda m(n)
\end{array}\right| \\
& +\cdots+\left|\begin{array}{cccc}
p(1)-\lambda m(1) & \cdots & 0 & 0 \\
-p(1) & \cdots & 0 & 0 \\
0 & \cdots & 0 & 0 \\
\vdots & \ddots & \vdots & \vdots \\
0 & \cdots & p(n-2)+p(n-1)-\lambda m(n-1) & -p(n-1) \\
0 & \cdots & 0 & -m(n)
\end{array}\right| .
\end{aligned}
$$

So, by simple computation, it follows that

$$
\begin{aligned}
Q_{n}^{\prime}(0)= & \left.\frac{d|J u-\lambda D u|}{d \lambda}\right|_{\lambda=0} \\
= & -m(1) p(1) p(2) \cdots p(n-1) \\
& -m(2) p(1) p(2) \cdots p(n-1) \\
& -\cdots-m(n) p(1) p(2) \cdots p(n-1) \\
= & -p(1) p(2) \cdots p(n-1) \sum_{s=1}^{n} m(s) .
\end{aligned}
$$

(2) If $\sum_{s=1}^{n} m(s)=0$, then $Q_{n}(0)=Q_{n}^{\prime}(0)=0$. Moreover, let $j_{1}, \ldots, j_{n-2}$ be $n-2$ elements in $\{1,2, \ldots, n-1\}$ with

$$
j_{1}<j_{2}<\cdots<j_{n-3}<j_{n-2}
$$

Let

$$
\begin{gathered}
\Lambda_{n}=\left\{\left(j_{1}, j_{2}, \ldots, j_{n-2}\right) \mid j_{k} \in\{1,2, \ldots, n-1\}\right. \\
\text { with } \left.j_{1}<j_{2}<\cdots<j_{n-3}<j_{n-2}\right\} .
\end{gathered}
$$

Let us arrange the elements of $\Lambda_{n}$ in the increasing order,

$$
\begin{aligned}
& (1,2, \ldots, n-3, n-2),(1,2, \ldots, n-3, n-1), \ldots, \\
& (2,3, \ldots, n-2, n-1) .
\end{aligned}
$$

It is easy to check that the number of the elements on $\Lambda_{n}$ is $n-$ 1 . We denote the $k$ th elements in (25) by $\beta_{k}, k=1,2, \ldots, n-1$. For given $\beta_{k}=\left(j_{1}, j_{2}, \ldots, j_{n-2}\right)$, define $a_{k}=P_{j_{1}} P_{j_{2}} \cdots P_{j_{n-2}}$. 
By computing and simplifying, we get that

$$
\begin{aligned}
\left.Q_{n}^{\prime \prime}(\lambda)\right|_{\lambda=0} & \\
= & \sum_{\substack{i, j=1 \\
i<j}}^{n} m(i) m(j) W_{n-2}(\ldots, i-1, i+1, \ldots, j-1, j+1, \ldots) \\
= & -2 a_{1}[m(1)+m(2)+\cdots+m(n-1)]^{2} \\
& -2 a_{2}[m(1)+m(2)+\cdots+m(n-2)]^{2}-\ldots \\
& -2 a_{n-2}[m(1)+m(2)]^{2}-2 a_{n-1} m(1)^{2} \\
= & -2 \sum_{k=1}^{n-1} a_{k}\left(\sum_{s=1}^{n-k} m(s)\right)^{2}
\end{aligned}
$$

and here $W_{n-2}(\ldots, i-1, i+1, \ldots, j-1, j+1, \ldots)$ denote the principal minor determinant of order $n-2$ of $J-\lambda D$, which is obtained by deleting the elements of $i$ column and $i$ row and deleting the elements of $j$ column and $j$ row. Thus, from (A0) and $\sum_{s=1}^{n} m(s)=0$, it follows that

$$
Q_{n}^{\prime \prime}(0)<0 \text {. }
$$

Lemma 9. For $j=1, \ldots, n-1$, the roots of $Q_{j}(\lambda)=0$ are simple. Moreover, one has the following.

(i) The largest negative root $\lambda_{j, 1}^{-}$and the smallest positive root $\lambda_{j, 1}^{+}$of $Q_{j}(\lambda)=0$ and the largest negative root $\lambda_{j+1,1}^{-}$and the smallest positive root $\lambda_{j+1,1}^{+}$of $Q_{j+1}(\lambda)=$ 0 satisfy

$$
\left(\lambda_{j, 1}^{-}, \lambda_{j, 1}^{+}\right) \supset\left(\lambda_{j+1,1}^{-}, \lambda_{j+1,1}^{+}\right), \quad j=2, \ldots, n .
$$

(ii) For $j=1, \ldots, n-1$, the positive roots of $Q_{j}(\lambda)=0$ and $Q_{j+1}(\lambda)=0$ separate one another; the negative roots of $Q_{j}(\lambda)=0$ and $Q_{j+1}(\lambda)=0$ separate one another.

(iii) If $\sum_{s=1}^{n} m(s) \neq 0$, then the roots of $Q_{n}(\lambda)=0$ are simple and 0 is a simple root; if $\sum_{s=1}^{n} m(s)=0$, then 0 is a double roots of $Q_{n}(\lambda)=0$ and the other root are simple.

Proof. First, we deal with the case $j=1$.

Obviously, $Q_{1}(\lambda)=c(1)-\lambda m(1)$. If $m(1)>0$, then

$$
\begin{gathered}
j=1, \quad j^{+}=1, \quad j^{-}=0, \\
\lambda_{1,1}^{+}=\frac{c(1)}{m(1)}>0 .
\end{gathered}
$$

If $m(1)<0$, then

$$
\begin{gathered}
j=1, \quad j^{+}=0, \quad j^{-}=1, \\
\lambda_{1,1}^{-}=\frac{c(1)}{m(1)}<0 .
\end{gathered}
$$

Recall $Q_{2}(\lambda)=(c(2)-\lambda m(2))(c(1)-\lambda m(1))-p^{2}(1)$. If $m(1)>0, m(2)>0$, then

$$
j=2, \quad j^{+}=2, \quad j^{-}=0,
$$

and $Q_{2}(\lambda)=0$ has two different roots $\lambda_{2,1}^{+}$and $\lambda_{2,2}^{+}$as follows:

$$
\begin{aligned}
\lambda_{2,1}^{+}= & (c(1) m(2)+c(2) m(1) \\
& \left.-\sqrt{(c(1) m(2)-c(2) m(1))^{2}+4 p^{2}(1) m(1) m(2)}\right) \\
& \times(2 m(1) m(2))^{-1}>0, \\
\lambda_{2,2}^{+}= & (c(1) m(2)+c(2) m(1) \\
& \left.+\sqrt{(c(1) m(2)-c(2) m(1))^{2}+4 p^{2}(1) m(1) m(2)}\right) \\
& \times(2 m(1) m(2))^{-1}>0 .
\end{aligned}
$$

It is easy to see that $0<\lambda_{2,1}^{+}<\lambda_{1,1}^{+}<\lambda_{2,2}^{+}$.

If $m(1)<0, m(2)>0$, then

$$
j=2, \quad j^{+}=1, \quad j^{-}=1,
$$

and $Q_{2}(\lambda)=0$ has two different roots $\lambda_{2,1}^{+}$and $\lambda_{2,1}^{-}$as follows:

$$
\begin{aligned}
\lambda_{2,1}^{+}= & (c(1) m(2)+c(2) m(1) \\
& \left.-\sqrt{(c(1) m(2)-c(2) m(1))^{2}+4 p^{2}(1) m(1) m(2)}\right) \\
& \times(2 m(1) m(2))^{-1}>0, \\
\lambda_{2,1}^{-}= & (c(1) m(2)+c(2) m(1) \\
& \left.+\sqrt{(c(1) m(2)-c(2) m(1))^{2}+4 p^{2}(1) m(1) m(2)}\right) \\
& \times(2 m(1) m(2))^{-1}<0 .
\end{aligned}
$$

It is easy to see that $\lambda_{1,1}^{-}<\lambda_{2,1}^{-}<0<\lambda_{2,1}^{+}$.

If $m(1)>0, m(2)<0$, then

$$
j=2, \quad j^{+}=1, \quad j^{-}=1,
$$

and $Q_{2}(\lambda)=0$ has two different roots $\lambda_{2,1}^{+}$and $\lambda_{2,1}^{-}$as follows:

$$
\begin{aligned}
\lambda_{2,1}^{+}= & (c(1) m(2)+c(2) m(1) \\
& \left.-\sqrt{(c(1) m(2)-c(2) m(1))^{2}+4 p^{2}(1) m(1) m(2)}\right) \\
& \times(2 m(1) m(2))^{-1}>0,
\end{aligned}
$$

$\lambda_{2,1}^{-}=(c(1) m(2)+c(2) m(1)$

$$
\begin{aligned}
& \left.+\sqrt{(c(1) m(2)-c(2) m(1))^{2}+4 p^{2}(1) m(1) m(2)}\right) \\
& \times(2 m(1) m(2))^{-1}<0 .
\end{aligned}
$$

It is easy to see that $\lambda_{1,1}^{-}<\lambda_{2,1}^{-}<0<\lambda_{2,1}^{+}$. 
If $m(1)<0, m(2)<0$, then

$$
j=2, \quad j^{+}=0, \quad j^{-}=2,
$$

and $Q_{2}(\lambda)=0$ has two different roots $\lambda_{2,1}^{-}$and $\lambda_{2,2}^{-}$as follows:

$$
\begin{aligned}
\lambda_{2,1}^{-}= & (c(1) m(2)+c(2) m(1) \\
& \left.+\sqrt{(c(1) m(2)-c(2) m(1))^{2}+4 p^{2}(1) m(1) m(2)}\right) \\
& \times(2 m(1) m(2))^{-1}<0, \\
\lambda_{2,2}^{-}= & (c(1) m(2)+c(2) m(1) \\
& \left.-\sqrt{(c(1) m(2)-c(2) m(1))^{2}+4 p^{2}(1) m(1) m(2)}\right) \\
& \times(2 m(1) m(2))^{-1}<0 .
\end{aligned}
$$

It is easy to see that $\lambda_{2,2}^{-}<\lambda_{1,1}^{-}<\lambda_{2,1}^{-}<0$. Thus, the assertion is true for $j=1$.

Second, suppose that for $j=k(k \leq n-3)$, and the relations of $Q_{k}(\lambda)=0$ and $Q_{k+1}(\lambda)=0$ are true, that is, the following two assertions hold.

If $m(k+1)>0$, then $(k+1)^{+}=k^{+}+1,(k+1)^{-}=k^{-}$and accordingly,

$$
\begin{gathered}
\lambda_{k, k^{-}}^{-}<\lambda_{k+1, k^{-}}^{-}<\cdots<\lambda_{k, 1}^{-}<\lambda_{k+1,1}^{-}<0 \\
0<\lambda_{k+1,1}^{+}<\lambda_{k, 1}^{+}<\cdots<\lambda_{k+1, k^{+}}^{+}<\lambda_{k, k^{+}}^{+}<\lambda_{k+1,(k+1)^{+}}^{+}
\end{gathered}
$$

If $m(k+1)<0$, then $(k+1)^{+}=k^{+},(k+1)^{-}=k^{-}+1$ and accordingly,

$$
\begin{gathered}
\lambda_{k+1, k^{-}+1}^{-}<\lambda_{k, k^{-}}^{-}<\lambda_{k+1, k^{-}}^{-}<\cdots<\lambda_{k, 1}^{-}<\lambda_{k+1,1}^{-}<0, \\
0<\lambda_{k+1,1}^{+}<\lambda_{k, 1}^{+}<\cdots<\lambda_{k+1, k^{+}}^{+}<\lambda_{k, k^{+}}^{+} .
\end{gathered}
$$

Now, we consider the case $j=k+1$. It is enough to verify the following four cases.

Case 1. $m(k+1)>0$ and $m(k+2)>0$.

In this case, $(k+2)^{+}=k^{+}+2,(k+2)^{-}=k^{-}$, we need to prove that

$$
\begin{gathered}
\lambda_{k+1, k^{-}}^{-}<\lambda_{k+2, k^{-}}^{-}<\cdots<\lambda_{k+1,1}^{-}<\lambda_{k+2,1}^{-}<0 \\
0<\lambda_{k+2,1}^{+}<\lambda_{k+1,1}^{+}<\cdots<\lambda_{k+2, k^{+}+1}^{+}<\lambda_{k+1, k^{+}+1}^{+}<\lambda_{k+2, k^{+}+2}^{+}
\end{gathered}
$$

Case 2. $m(k+1)>0$ and $m(k+2)<0$.

In this case, $(k+2)^{+}=k^{+}+1,(k+2)^{-}=k^{-}+1$, we need to prove that

$$
\begin{gathered}
\lambda_{k+2, k^{-}+1}^{-}<\lambda_{k+1, k^{-}}^{-}<\lambda_{k+2, k^{-}}^{-}<\cdots<\lambda_{k+1,1}^{-}<\lambda_{k+2,1}^{-}<0, \\
0<\lambda_{k+2,1}^{+}<\lambda_{k+1,1}^{+}<\cdots<\lambda_{k+2, k^{+}+1}^{+}<\lambda_{k+1, k^{+}+1}^{+} .
\end{gathered}
$$

Case 3. $m(k+1)<0$ and $m(k+2)<0$.

In this case, $(k+2)^{+}=k^{+},(k+2)^{-}=k^{-}+2$, we need to prove that

$$
\begin{aligned}
\lambda_{k+2, k^{-}+2}^{-} & <\lambda_{k+1, k^{-}+1}^{-}<\lambda_{k+2, k^{-}+1}^{-}<\cdots<\lambda_{k+1,1}^{-}<\lambda_{k+2,1}^{-}<0 \\
0 & <\lambda_{k+2,1}^{+}<\lambda_{k+1,1}^{+}<\cdots<\lambda_{k+2, k^{+}}^{+}<\lambda_{k+1, k^{+}}^{+} .
\end{aligned}
$$

Case 4. $m(k+1)<0$ and $m(k+2)>0$.

In this case, $(k+2)^{+}=k^{+}+1,(k+2)^{-}=k^{-}+1$, we need to prove that

$$
\begin{aligned}
& \lambda_{k+2, k^{-}+1}^{-}<\lambda_{k+1, k^{-}}^{-}<\lambda_{k+2, k^{-}}^{-}<\cdots<\lambda_{k+1,1}^{-}<\lambda_{k+2,1}^{-}<0, \\
& 0<\lambda_{k+2,1}^{+}<\lambda_{k+1,1}^{+}<\cdots<\lambda_{k+2, k^{+}}^{+}<\lambda_{k+1, k^{+}}^{+}<\lambda_{k+2, k^{+}+1}^{+} .
\end{aligned}
$$

We only deal with Case 1 . The other cases could be dealt via the same method.

First, we show that (42) holds. that

Since $(k+2)^{-}=(k+1)^{-}=k^{-}$, it follows from Lemma 4

$$
\begin{gathered}
(-1)^{k^{-}} Q_{k}(-\infty)>0 \\
(-1)^{(k+1)^{-}} Q_{k+1}(-\infty)=(-1)^{k^{-}} Q_{k+1}(-\infty)>0 .
\end{gathered}
$$

We only deal with the case that $k^{-}$is even. The case $k^{-}$is odd could be treated by the same way.

Thus (47) reduces to

$$
\begin{gathered}
Q_{k}(-\infty)>0, \\
Q_{k+1}(-\infty)>0 .
\end{gathered}
$$

Recall (39) as follows:

$$
\lambda_{k, k^{-}}^{-}<\lambda_{k+1, k^{-}}^{-}<\lambda_{k, k^{-}-1}^{-}<\lambda_{k+1, k^{-}-1}^{-}<\cdots<\lambda_{k, 1}^{-}<\lambda_{k+1,1}^{-}<0
$$

and the fact that

$$
Q_{k+1}\left(\lambda_{k+1, k^{-}}^{-}\right)=Q_{k+1}\left(\lambda_{k+1, k^{-}-1}^{-}\right)=\cdots=Q_{k+1}\left(\lambda_{k+1,1}^{-}\right)=0 .
$$

It follows from (47), (48), and (49) that

$$
(-1)^{j} Q_{k}\left(\lambda_{k+1, k^{-}-j}^{-}\right)<0, \quad j=0, \ldots, k^{-}-1 .
$$

Combining this with (50) and using Lemma 7, it concludes that

$$
(-1)^{j} Q_{k+2}\left(\lambda_{k+1, k^{-}-j}^{-}\right)>0, \quad j=0, \ldots, k^{-}-1 .
$$

In particular,

$$
Q_{k+2}\left(\lambda_{k+1,1}^{-}\right)<0 .
$$

By Lemma $5, Q_{k+2}(\lambda)=0$ has exactly $k^{-}$zeros in $(-\infty, 0)$. This together with (52), (53), and the fact that

$$
Q_{k}(0)>0, \quad Q_{k+1}(0)>0, \quad Q_{k+2}(0)>0
$$


implies that there exist $\lambda_{k+2, k^{-}-j}^{-} \in\left(\lambda_{k+1, k^{-}-j}^{-}, \lambda_{k+1, k^{-}-j-1}^{-}\right), j=$ $0, \ldots, k^{-}-2$, and $\lambda_{k+2,1}^{-} \in\left(\lambda_{k+1,1}^{-}, 0\right)$, such that

$$
Q_{k+2}\left(\lambda_{k+2, k^{-}-j}^{-}\right)=0, \quad j=0, \ldots, k^{-}-1 .
$$

Therefore, (42) is valid.

Next, we show that (43) is true.

Obviously, $m(k+1)>0, m(k+2)>0$ yields

$$
(k+2)^{+}=(k+1)^{+}+1=k^{+}+2 .
$$

In the following, we only deal with the case that $k^{+}$is even. The case $k^{+}$is odd could be treated by the same way.

From Lemma 4, we have that

$$
Q_{k}(+\infty)>0, \quad Q_{k+1}(+\infty)<0, \quad Q_{k+2}(+\infty)>0 .
$$

Combining this with (40) as

$$
0<\lambda_{k+1,1}^{+}<\lambda_{k, 1}^{+}<\cdots<\lambda_{k+1, k^{+}}^{+}<\lambda_{k, k^{+}}^{+}<\lambda_{k+1,(k+1)^{+}}^{+},
$$

and using the fact that

$$
Q\left(\lambda_{k+1,(k+1)^{+}-j}^{+}\right)=0, \quad j=0, \ldots,(k+1)^{+}-1,
$$

it concludes that

$$
(-1)^{j} Q_{k}\left(\lambda_{k+1,(k+1)^{+}-j}^{+}\right)>0, \quad j=0, \ldots,(k+1)^{+}-1 .
$$

This together with Lemma 7 implies that

$$
(-1)^{j} Q_{k+2}\left(\lambda_{k+1,(k+1)^{+}-j}^{+}\right)<0, \quad j=0, \ldots,(k+1)^{+}-1 .
$$

In particular, for $j=0$,

$$
Q_{k+2}\left(\lambda_{k+1,(k+1)^{+}}^{+}\right)<0 .
$$

This together with the third inequality in (57) implies that

$$
Q_{k+2}\left(\lambda_{k+2,(k+1)^{+}+1}\right)=0
$$

for some $\lambda_{k+2,(k+1)^{+}+1} \in\left(\lambda_{k+1,(k+1)^{+}}^{+}, \infty\right)$.

Using (61) with $j=(k+1)^{+}-1$, we get

$$
Q_{k+2}\left(\lambda_{k+1,1}^{+}\right)<0,
$$

which together with the fact

$$
Q_{k+2}(0)>0
$$

implies that

$$
Q_{k+2}\left(\lambda_{k+2,1}^{+}\right)=0
$$

for some $\lambda_{k+2,1}^{+} \in\left(0, \lambda_{k+1,1}^{+}\right)$.
Now, for $j=1, \ldots,(k+1)^{+}-1$, there exist $\lambda_{k+2,(k+2)^{+}-j}^{+} \epsilon$ $\left(\lambda_{k+1,(k+1)^{+}-j}^{+}, \lambda_{k+1,(k+1)^{+}-j+1}^{+}\right)$, such that

$$
Q_{k+2}\left(\lambda_{k+2,(k+2)^{+}-j}^{+}\right)=0, \quad j=0, \ldots,(k+1)^{+} .
$$

Therefore, (43) is valid.

Finally, for $j=n$, the relation $Q_{n}(\lambda)=0$ and $Q_{n-1}(\lambda)=0$ are also true. From above conclusions, we have $Q_{n-1}(\lambda)=$ 0 has $(n-1)^{-}$negative roots and $(n-1)^{+}$positive roots satisfying

$$
\begin{aligned}
\lambda_{n-1,(n-1)^{-}}^{-} & <\lambda_{n-1,(n-1)^{-}-1}^{-}<\cdots<\lambda_{n-1,1}^{-} \\
& <0<\lambda_{n-1,1}^{+}<\lambda_{n-1,2}^{+}<\cdots<\lambda_{n-1,(n-1)^{+}}^{+}
\end{aligned}
$$

If $m(n)>0$, we have that $n^{+}=(n-1)^{+}+1, n^{-}=(n-1)^{-}$. By a similar argument and together with the fact $Q_{n}(0)=0$ and Lemma 8, it follows that

(i) if $\sum_{s=0}^{n} m(s)<0$, then

$$
\begin{aligned}
\lambda_{n-1,(n-1)^{-}}^{-} & <\lambda_{n, n^{-}}^{-}<\cdots<\lambda_{n-1,1}^{-}<\lambda_{n, 1}^{-} \\
& =0<\lambda_{n, 1}^{+}<\lambda_{n-1,1}^{+}<\cdots<\lambda_{n-1,(n-1)^{+}}^{+}<\lambda_{n, n^{+}}^{+} ;
\end{aligned}
$$

(ii) if $\sum_{s=0}^{n} m(s)>0$, then

$$
\begin{aligned}
\lambda_{n-1,(n-1)^{-}}^{-} & <\lambda_{n, n^{-}}^{-}<\cdots<\lambda_{n-1,1}^{-}<\lambda_{n, 1}^{-}<0 \\
& =\lambda_{n, 1}^{+}<\lambda_{n-1,1}^{+}<\lambda_{n, 2}^{+}<\cdots<\lambda_{n-1,(n-1)^{+}}^{+}<\lambda_{n, n^{+}}^{+} ;
\end{aligned}
$$

(iii) if $\sum_{s=0}^{n} m(s)=0$, then

$$
\begin{aligned}
\lambda_{n-1,(n-1)^{-}}^{-} & <\lambda_{n, n^{-}}^{-}<\cdots<\lambda_{n-1,1}^{-}<\lambda_{n, 1}^{-} \\
& =0=\lambda_{n, 1}^{-}<\lambda_{n-1,1}^{+}<\lambda_{n, 2}^{+} \\
& <\cdots<\lambda_{n-1,(n-1)^{+}}^{+}<\lambda_{n, n^{+}}^{+}
\end{aligned}
$$

If $m(n)<0$, we have that $n^{+}=(n-1)^{+}, n^{-}=(n-1)^{-}+1$. By a similar method and together with $Q_{n}(0)=0$ and Lemma 8, we get that

(i) if $\sum_{s=0}^{n} m(s)<0$, then

$$
\begin{aligned}
\lambda_{n, n^{-}}^{-} & <\lambda_{n-1,(n-1)^{-}}^{-}<\cdots<\lambda_{n-1,1}^{-}<\lambda_{n, 1}^{-} \\
& =0<\lambda_{n, 1}^{+}<\lambda_{n-1,1}^{+}<\cdots<\lambda_{n, n^{+}}^{+}<\lambda_{n-1,(n-1)^{+}}^{+}
\end{aligned}
$$

(ii) if $\sum_{s=0}^{n} m(s)>0$, then

$$
\begin{aligned}
\lambda_{n, n^{-}}^{-} & <\lambda_{n-1,(n-1)^{-}}^{-}<\cdots<\lambda_{n-1,1}^{-}<\lambda_{n, 1}^{-}<0 \\
& =\lambda_{n, 1}^{+}<\lambda_{n-1,1}^{+}<\lambda_{n, 2}^{+}<\cdots<\lambda_{n, n^{+}}^{+}<\lambda_{n-1,(n-1)^{+}}^{+}
\end{aligned}
$$


(iii) if $\sum_{s=0}^{n} m(s)=0$, then

$$
\begin{aligned}
\lambda_{n, n^{-}}^{-} & <\lambda_{n-1,(n-1)^{-}}^{-}<\cdots<\lambda_{n, 2}^{-}<\lambda_{n-1,1}^{-}<\lambda_{n, 1}^{-} \\
& =0=\lambda_{n, 1}^{+}<\lambda_{n-1,1}^{+}<\cdots<\lambda_{n, n^{+}}^{+}<\lambda_{n-1,(n-1)^{+}}^{+}
\end{aligned}
$$

Thus the proof is complete.

Lemma 10. Let $w(\lambda)$ be the number of sign changes in the sequence (15). Then for $i \in\left\{1, \ldots, n^{+}\right\}$,

$$
\lim _{\lambda \rightarrow\left(\lambda_{n, i}^{+}\right)^{-}} w(\lambda)=i-1, \quad \lim _{\lambda \rightarrow\left(\lambda_{n, i}^{+}\right)^{+}} w(\lambda)=i
$$

where $\lambda \rightarrow(a)^{-}$means that $\lambda \rightarrow a$ in $(-\infty, a)$ and $\lambda \rightarrow(a)^{+}$ means that $\lambda \rightarrow$ a in $(a, \infty)$.

Proof. It is motivated by the proof of Strums Theorem; see [20, Theorem 1.4.3] and its proof.

The idea of the proof is to follow the changes in $w$ as $\lambda$ passes through the interval $[0, n]$. In particular, we will show that $w$ is a monotonically increasing function and that each root of $Q_{n}$ and only a root of $Q_{n}$ make $w$ jump by 1 .

If $Q_{j}(\hat{\lambda})=0$ for some $j \in\{1, \ldots, n-1\}$, then for $Q_{j-1}$, $Q_{j}, Q_{j+1}$ we have from Lemma 7 that $Q_{j-1}$ and $Q_{j+1}$ have opposite, but constant signs, since $Q_{j-1}$ and $Q_{j+1}$ cannot be zero in a sufficiently small neighborhood $U(\widehat{\lambda})$ and thus cannot change sign. Hence, whatever the sign of $Q_{j}$ is in $U(\widehat{\lambda})$, it does not change the overall sign change count (to see this, note that $Q_{j-1}$ and $Q_{j+1}$ have opposite signs, and hence if the sign sequence before is +-- , it is after ++- and the number of sign changes remains the same. The same for the other cases). In other word, $w(\lambda)$ stays constant when $\lambda$ passing through a root of $Q_{j}$ from some $j \in\{1, \ldots, n-1\}$.

It is easy to see from Lemma 9 that

$$
\operatorname{sgn} Q_{n-1}\left(\lambda_{n, i}^{+}\right)=(-1)^{i-1}, \quad i \in\left\{1, \ldots, n^{+}\right\} .
$$

Next, we show that each root of $Q_{n}$ and only a root of $Q_{n}$ make $w$ jump by 1 .

In fact, for $i=1, Q_{n-1}\left(\lambda_{n, 1}^{+}\right)>0$, which implies that there exists a neighborhood $U\left(\lambda_{n, 1}^{+}\right)$of $\lambda_{n, 1}^{+}$, such that

$$
Q_{n-1}(\lambda)>0, \quad \lambda \in U\left(\lambda_{n, 1}^{+}\right)
$$

From the definition of $\lambda_{n, 1}^{+}$,

$$
Q_{n}(\lambda)>0, \quad \lambda \in\left[0, \lambda_{n, 1}^{+}\right)
$$

The chain of signs switches from "..++ " to “...+ -" when passing through $\lambda_{n, 1}^{+}$, so $w$ increases by 1 .

For $i=2$,

$$
\begin{gathered}
Q_{n-1}\left(\lambda_{n, 2}^{+}\right)<0, \\
Q_{n}(\lambda)<0, \quad \lambda \in\left(\lambda_{n, 1}^{+}, \lambda_{n, 2}^{+}\right) .
\end{gathered}
$$

The chain of signs switches from “...--" to “...-+" when passing through $\lambda_{n, 2}^{+}$, so $w$ increases by 1 .

Repeating the above argument, we may deduce that

$$
\lim _{\lambda \rightarrow\left(\lambda_{n, i}^{+}\right)^{-}} w(\lambda)=i-1, \quad \lim _{\lambda \rightarrow\left(\lambda_{n, i}^{+}\right)^{+}} w(\lambda)=i
$$

This completes the proof.

Lemma 11. If $u(\cdot, \lambda)$ satisfies (1), (2) with $u(1, \lambda)=1$, then

$$
u(k+1, \lambda)=p(1) \cdots p(k) Q_{k}(\lambda), \quad k=1, \ldots, n-1 .
$$

Proof. Let

$$
u=(u(0), u(1), u(2), \ldots, u(n), u(n+1))^{T}
$$

be a solution of (1), (2). Then

$$
\begin{gathered}
(c(1)-\lambda m(1)) u(1)-p(1) u(2)=0, \\
-p(1) u(1)+(c(2)-\lambda m(2)) u(2)-p(2) u(3)=0, \\
\ldots \\
-p(n-2) u(n-2)+(c(n-1)-\lambda m(n-1)) u(n-1) \\
-p(n-1) u(n)=0, \\
-p(n-1) u(n-1)+(c(n)-\lambda m(n)) u(n)=0 .
\end{gathered}
$$

Clearly, (83) is equivalent to

$$
\begin{aligned}
& -p(k-1) u(k-1)+(p(k-1)+p(k)-\lambda m(k)) u(k) \\
& -p(k) u(k+1)=0, \quad k=1, \ldots, n,
\end{aligned}
$$

with $u(0)$ and $u(n+1)$ determined by (2).

Let

$$
\begin{array}{r}
v_{0}=u(0), \quad v_{1}=u(1), \quad v_{k}=p(1) \cdots p(k-1) u(k), \\
k=2, \ldots n .
\end{array}
$$

Then

$$
\begin{array}{r}
v_{1}=u(1, \lambda)=1=Q_{0}(\lambda), \quad v_{2}=Q_{1}(\lambda), \\
v_{k+1}=(c(k)-\lambda m(k)) v_{k}-p^{2}(k-1) v_{k-1}, \\
k=2,3, \ldots n-1 .
\end{array}
$$

Obviously, $v_{k+1}$ and $Q_{k}(\lambda)$ satisfy the same recurrence formula (15), and it follows that

$$
v_{k+1}=Q_{k}(\lambda), \quad k=1, \ldots, n-1,
$$

and accordingly,

$$
Q_{k}(\lambda)=p(1) p(2) \cdots p(k) u(k+1, \lambda), \quad k=1, \ldots, n-1 .
$$


Proof of Theorem 1. From Lemmas 5 and 9, we can obtain the following consequence.

(i) If $\sum_{t=1}^{n} m(t)>0$, then (1), (2) has $n$ real and simple eigenvalues, which can be ordered as follows:

$$
\begin{aligned}
\lambda_{n^{-}}^{-} & <\lambda_{n^{-}-1}^{-}<\cdots<\lambda_{2}^{-}<\lambda_{1}^{-}<0 \\
& =\lambda_{1}^{+}<\lambda_{2}^{+}<\cdots<\lambda_{n^{+}}^{+}
\end{aligned}
$$

(ii) If $\sum_{t=1}^{n} m(t)<0$, then (1), (2) has $n$ real and simple eigenvalues, which can be ordered as follows:

$$
\begin{aligned}
\lambda_{n^{-}}^{-} & <\lambda_{n^{-}-1}^{-}<\cdots<\lambda_{2}^{-}<\lambda_{1}^{-} \\
& =0<\lambda_{1}^{+}<\lambda_{2}^{+}<\cdots<\lambda_{n^{+}}^{+}
\end{aligned}
$$

(iii) If $\sum_{t=1}^{n} m(t)=0$, then $\lambda=0$ is an eigenvalue of (1), (2) and other $n-2$ eigenvalues are real and simple, which can be ordered as follows:

$$
\begin{aligned}
\lambda_{n^{-}}^{-} & <\lambda_{n^{-}-1}^{-}<\cdots<\lambda_{2}^{-}<\lambda_{1}^{-} \\
& =0=\lambda_{1}^{+}<\lambda_{2}^{+}<\cdots<\lambda_{n^{+}}^{+}
\end{aligned}
$$

Now, we consider the numbers of sign changing of eigenfunction. From Lemma 11, we may determine that the number of sign changing of

$$
\{u(0), u(1), \ldots, u(n), u(n+1)\}
$$

via that of

$$
\left\{Q_{1}\left(\lambda_{n, i}^{+}\right), \ldots, Q_{n-1}\left(\lambda_{n, i}^{+}\right)\right\}
$$

since (2) implies that $u(0) u(1) \geq 0$ and $u(n) u(n+1) \geq 0$.

Let $\widehat{w}(\lambda)$ be the number of sign changes in the sequence

$$
\begin{gathered}
Q_{0}(\lambda)=1 ; \\
Q_{1}(\lambda)=c(1)-\lambda m(1) ; \\
Q_{j}(\lambda)=(c(j)-\lambda m(j)) Q_{j-1}(\lambda)-p^{2}(j-1) Q_{j-2}(\lambda), \\
j=2,3, \ldots, n-1 ; \\
Q_{n-1}(\lambda)=(c(n-1)-\lambda m(n-1)) \\
\times Q_{n-2}(\lambda)-p^{2}(n-2) Q_{n-3}(\lambda) .
\end{gathered}
$$

Using the same method to prove Lemma 10, with obvious changes, we may obtain that for $i \in\left\{2, \ldots,(n-1)^{+}\right\}$,

$$
\lim _{\lambda \rightarrow \lambda_{n-1, i-1}^{+}+0} \widehat{w}(\lambda)=i-1, \quad \lim _{\lambda \rightarrow \lambda_{n-1, i}^{+}-0} \widehat{w}(\lambda)=i-1 .
$$

Thus, for $i \in\left\{2, \ldots,(n-1)^{+}\right\}$, Lemma 9 yields

$$
\lambda_{n-1, i-1}^{+}<\lambda_{n, i}^{+}<\lambda_{n-1, i}^{+}
$$

This together with (95) and the fact that $\widehat{w}(\lambda)$ is nondecreasing in $(0, \infty)$ implies

$$
\widehat{w}\left(\lambda_{n, i}^{+}\right)=\widehat{w}\left(\frac{\lambda_{n-1, i-1}^{+}+\lambda_{n-1, i}^{+}}{2}\right)=i-1,
$$

and accordingly

$$
w\left(\lambda_{n, i}^{+}\right)=\widehat{w}\left(\lambda_{n, i}^{+}\right)=i-1 .
$$

For the case $i=1$, since $Q_{j}(0)>0$ for $j \in\{0,1, \ldots, n-1\}$, it follows that

$$
\lim _{\lambda \rightarrow 0^{+}} \widehat{w}(\lambda)=0
$$

This together with the facts $0 \leq \lambda_{n, 1}^{+}<\lambda_{n-1,1}^{+}$and

$$
\lim _{\lambda \rightarrow\left(\lambda_{n-1,1}^{+}\right)^{-}} \widehat{w}(\lambda)=0
$$

implies that

$$
w\left(\lambda_{n, 1}^{+}\right)=\widehat{w}\left(\lambda_{n, 1}^{+}\right)=0 .
$$

If $n^{+}=(n-1)^{+}$, then it has been done! If $n^{+}=(n-1)^{+}+1$, then by the same method, with obvious changes, we may get

$$
w\left(\lambda_{n, n^{+}}^{+}\right)=\widehat{w}\left(\lambda_{n, n^{+}}^{+}\right)=n^{+}-1
$$

Finally, by using the above method, with obvious changes, we may prove that the number of sign changes $\psi_{n, i}^{-}$is $i-1$.

Remark 12. If one extra term $q(t) y(t)$ is present in (1), then $\lambda=0$ is generally not an eigenvalue of

$$
\begin{gathered}
\Delta[p(t-1) \Delta u(t-1)]-q(t) u(t)+\lambda m(t) u(t)=0, \\
t \in \mathbb{T}, \\
u(0)=u(1), \quad u(n)=u(n+1),
\end{gathered}
$$

anymore. Recall the fact that $\lambda=0$ is essential in Lemma 8 and its proof. Therefore, the spectrum structure of the more general problem (103) is still open.

\section{Acknowledgments}

The authors are very grateful to the anonymous referees for their valuable suggestions. This study is supported by the NSFC (no. 11061030), SRFDP (no. 20126203110004) and Gansu Provincial National Science Foundation of China (no. 1208RJZA258).

\section{References}

[1] F. V. Atkinson, Discrete and Continuous Boundary Problems, Mathematics in Science and Engineering, Vol. 8, Academic Press, New York, NY, USA, 1964. 
[2] A. Jirari, "Second-order Sturm-Liouville difference equations and orthogonal polynomials," Memoirs of the American Mathematical Society, vol. 113, no. 542, pp. 1-138, 1995.

[3] W. G. Kelley and A. C. Peterson, Difference Equations: An Introduction with Applications, Academic Press, Boston, Mass, USA, 1991.

[4] R. P. Agarwal, M. Bohner, and P. J. Y. Wong, "Sturm-Liouville eigenvalue problems on time scales," Applied Mathematics and Computation, vol. 99, no. 2-3, pp. 153-166, 1999.

[5] Y. Shi and S. Chen, "Spectral theory of second-order vector difference equations," Journal of Mathematical Analysis and Applications, vol. 239, no. 2, pp. 195-212, 1999.

[6] M. Bohner, "Discrete linear Hamiltonian eigenvalue problems," Computers \& Mathematics with Applications, vol. 36, no. 10-12, pp. 179-192, 1998.

[7] E. L. Ince, Ordinary Differential Equations, Dover Publications, New York, NY, USA, 1926.

[8] P. Hess and T. Kato, "On some linear and nonlinear eigenvalue problems with an indefinite weight function," Communications in Partial Differential Equations, vol. 5, no. 10, pp. 999-1030, 1980.

[9] A. Anane, O. Chakrone, and M. Moussa, "Spectrum of one dimensional $p$-Laplacian operator with indefinite weight," Electronic Journal of Qualitative Theory of Differential Equations, vol. 2002, no. 17, pp. 1-11, 2002.

[10] M. Zhang, "Nonuniform nonresonance of semilinear differential equations," Journal of Differential Equations, vol. 166, no. 1, pp. 33-50, 2000.

[11] R. Ma, J. Xu, and X. Han, "Global bifurcation of positive solutions of a second-order periodic boundary value problems with indefinite weight," Nonlinear Analysis: Theory, Methods \& Applications, vol. 71, no. 5-6, pp. 2119-2125, 2009.

[12] R. Ma and C. Gao, "Sign-changing solutions of nonlinear boundary value problems of difference equations," Indian Journal of Pure and Applied Mathematics, vol. 39, no. 4, pp. 323-332, 2008.

[13] K. J. Brown, "Local and global bifurcation results for a semilinear boundary value problem," Journal of Differential Equations, vol. 239, no. 2, pp. 296-310, 2007.

[14] J. Ji and B. Yang, "Eigenvalue comparisons for a class of boundary value problems of second order difference equations," Linear Algebra and Its Applications, vol. 420, no. 1, pp. 218-227, 2007.

[15] J. Ji and B. Yang, "Eigenvalue comparisons for second order difference equations with Neumann boundary conditions," Linear Algebra and Its Applications, vol. 425, no. 1, pp. 171-183, 2007.

[16] R. Ma and C. Gao, "Bifurcation of positive solutions of a nonlinear discrete fourth-order boundary value problem," Zeitschrift für Angewandte Mathematik und Physik, vol. 64, no. 3, pp. 493506, 2013.

[17] F. R. Gantmaher and M. G. Kreĭn, Oscillation Matrices and Kernels and Small Oscillations of Mechanical Systems, Gosudarstvennym Izdatel'stvom Tehniko-Teoreticheskoj Literatury, Moscow, Russia, 1950.

[18] R. A. Horn and C. R. Johnson, Matrix Analysis, Cambridge University Press, Cambridge, Mass, USA, 1990.

[19] F. R. Gantmacher, The Theory of Matrices. Vol. 1, AMS Chelsea Publishing, New York, NY, USA, 1960.
[20] V. V. Prasolov, Polynomials, vol. 11 of Algorithms and Computation in Mathematics, Springer, Berlin, Germany, 2010, Translated from the 2001 Russian second edition by Dimitry Leites. 


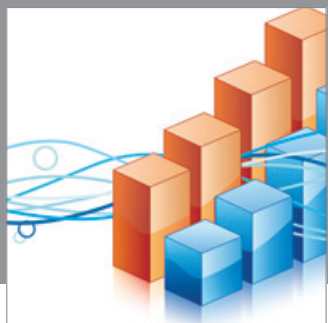

Advances in

Operations Research

mansans

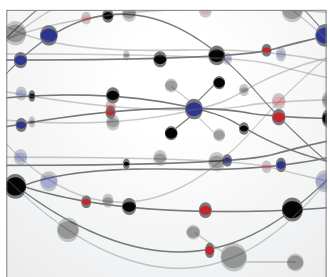

The Scientific World Journal
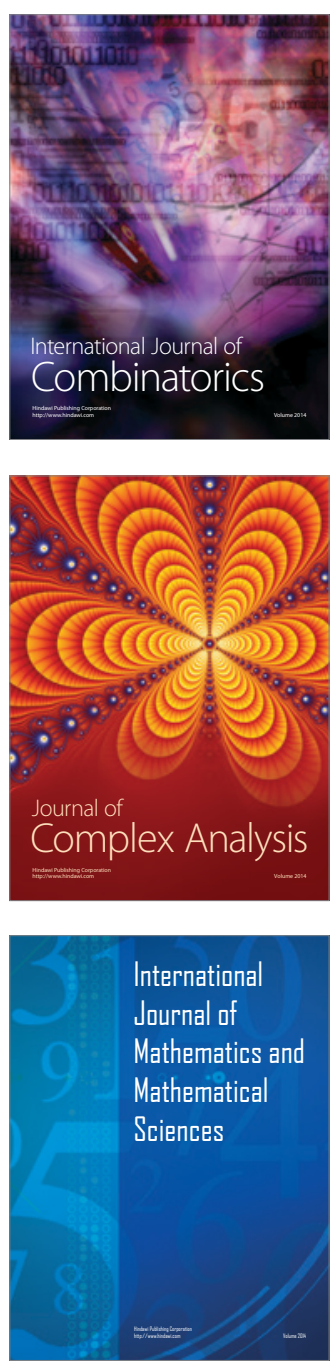
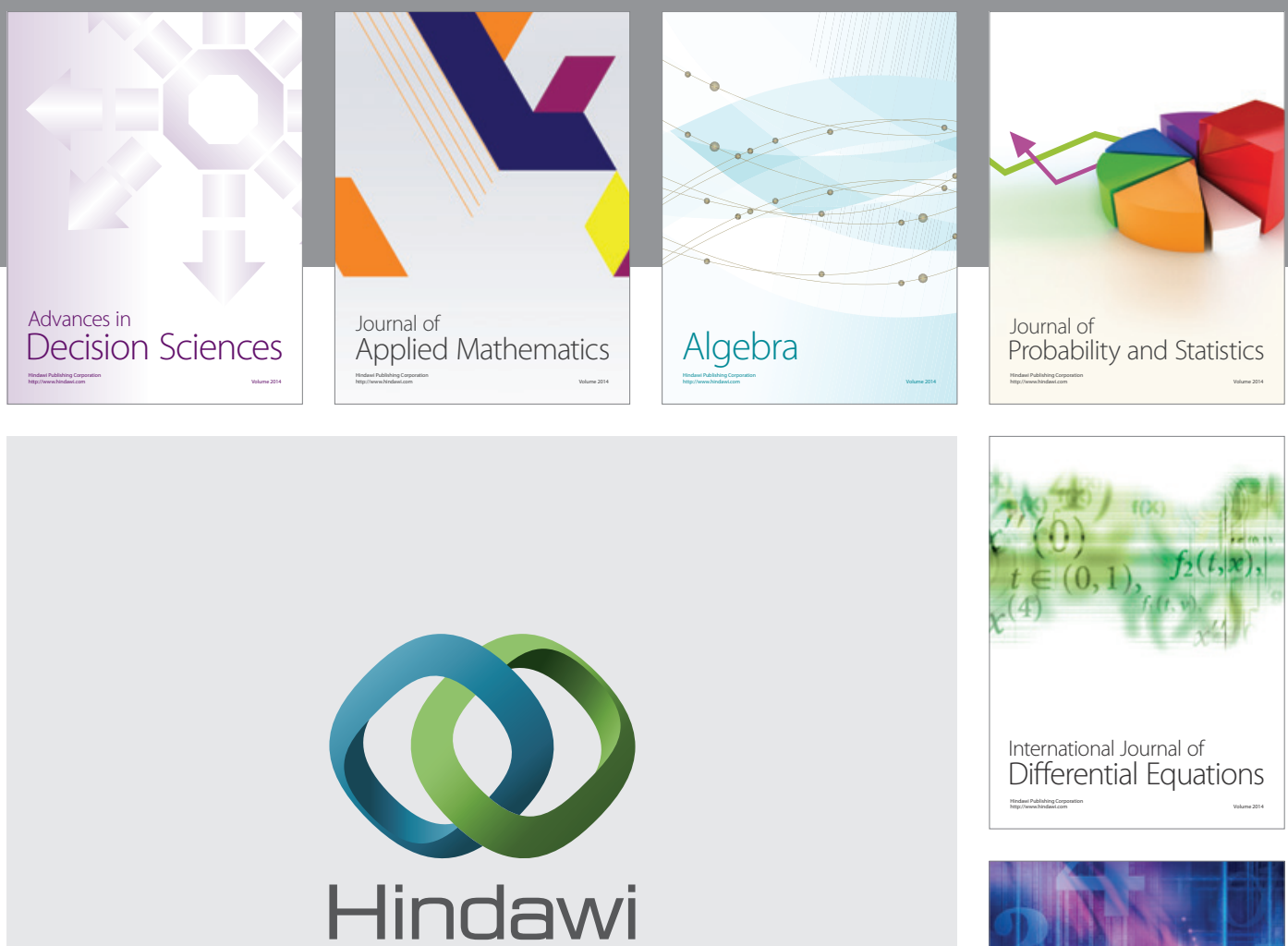

Submit your manuscripts at http://www.hindawi.com
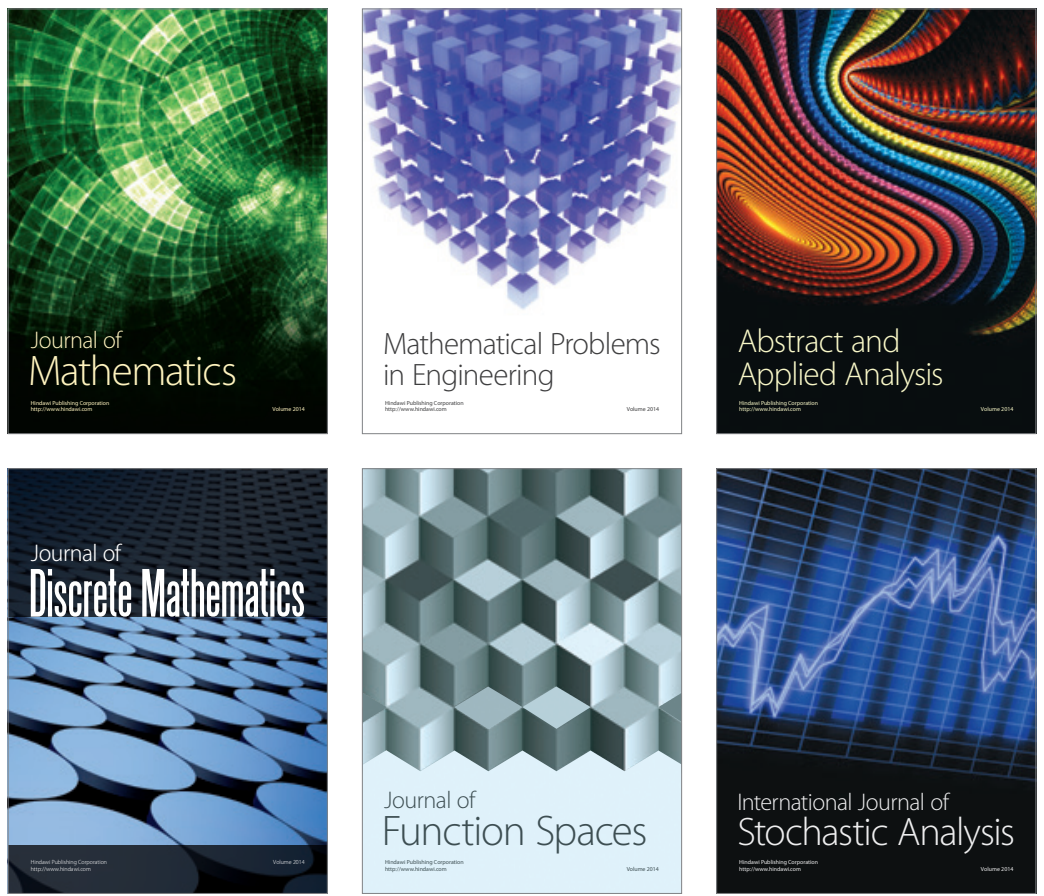

Journal of

Function Spaces

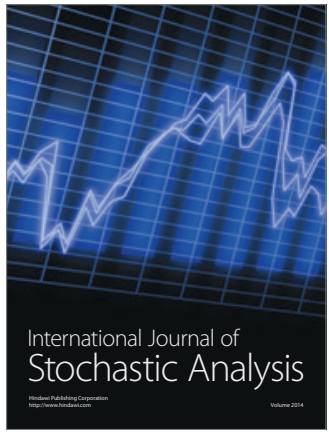

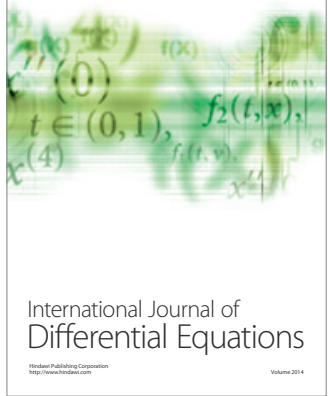
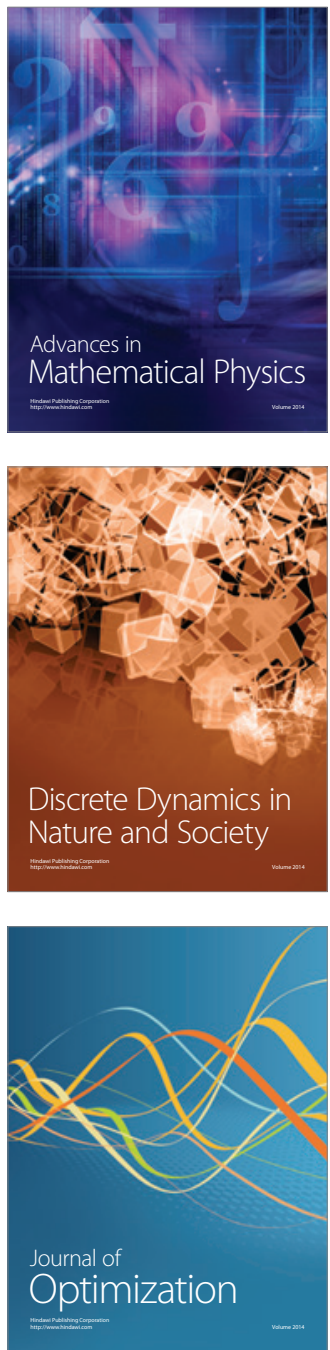